Article

\title{
New Insights into the Virulence Traits and Antibiotic Resistance of Enterococci Isolated from Diverse Probiotic Products
}

\author{
Fengru Deng ${ }^{1,2,3}$, Yunsheng Chen ${ }^{1,2,3}$, Xiaoyu Zhou ${ }^{1,2,3}$, Huiying Xiao ${ }^{1,2,3}$, Tianyu Sun $1,2,3$, Yiqun Deng $1,2,3, * \mathbb{D}$ \\ and Jikai Wen $1,2,3, *$
}

1 Guangdong Provincial Key Laboratory of Protein Function and Regulation in Agricultural Organisms, College of Life Sciences, South China Agricultural University, Guangzhou 510642, Guangdong, China; dengfengru@scau.edu.cn (F.D.); yunshengchen@stu.scau.edu.cn (Y.C.); 20202003036@stu.scau.edu.cn (X.Z.); huiyingxiao@stu.scau.edu.cn (H.X.); suntianyu@stu.scau.edu.cn (T.S.)

2 Key Laboratory of Zoonosis of Ministry of Agriculture and Rural Affairs, South China Agricultural University, Guangzhou 510642, Guangdong, China

3 Guangdong Laboratory for Lingnan Modern Agriculture, South China Agricultural University, Guangzhou 510642, Guangdong, China

* Correspondence: yqdeng@scau.edu.cn (Y.D.); jkwen@scau.edu.cn (J.W.)

Citation: Deng, F.; Chen, Y.; Zhou, X.; Xiao, H.; Sun, T.; Deng, Y.; Wen, J. New Insights into the Virulence Traits and Antibiotic Resistance of Enterococci Isolated from Diverse Probiotic Products. Microorganisms 2021, 9, 726. https://doi.org/ 10.3390/microorganisms 9040726

Academic Editor: Nathalie Connil

Received: 23 February 2021

Accepted: 26 March 2021

Published: 31 March 2021

Publisher's Note: MDPI stays neutral with regard to jurisdictional claims in published maps and institutional affiliations.

Copyright: (c) 2021 by the authors. Licensee MDPI, Basel, Switzerland. This article is an open access article distributed under the terms and conditions of the Creative Commons Attribution (CC BY) license (https:/ / creativecommons.org/licenses/by/ $4.0 /)$.
Abstract: The GRAS (generally recognized as safe) status of Enterococcus has not yet been authenticated, but enterococci, as probiotics, have been increasingly applied in human healthcare and animal husbandry, for instance as a dietary supplement, feed additive, or growth promotor. The food chain is the important route for introducing enterococci into the human gut. The pathogenicity of Enterococcus from probiotic products requires investigation. In the study, 110 commercial probiotic products used for human, animal, aquaculture, and plants were examined, among which 36 enterococci were identified, including 31 from Enterococcus faecium, 2 from E. faecalis, 2 from E. casseliflavus, and 1 from E. gallinarum. Strikingly, 28 of the 36 enterococci isolated from probiotics here did not mention the presence of Enterococcus in the labeled ingredients, and no Enterococcus isolates were found from 5 animal probiotics that were labeled with the genus. In total, 35 of the 110 products exhibited hemolysis, including 5 (10.6\%) human probiotics, 14 (41.2\%) animal probiotics, 8 (57.1\%) aquaculture probiotics, and 8 (53.3\%) plant probiotics. The detection rates of virulence factors associated with adhesion, antiphagocytosis, exoenzyme, biofilm, and other putative virulence markers (PVM) in 36 enterococci were $94.4 \%, 91.7 \%, 5.6 \%, 94.4 \%$ and $8.3 \%$. Twenty-six of the 36 isolated strains exhibited biofilm formation ability, where 25 strains $(69.4 \%)$ and one $(2.8 \%)$ were strong and weak biofilm producers, respectively. We analyzed the resistance rates against erythromycin (97\%), vancomycin and ciprofloxacin $(8 \%)$, tetracycline $(3 \%)$, and high-level aminoglycosides $(0 \%)$, respectively. High detection rates of $\mathrm{msrC} / \mathrm{ls} a \mathrm{~A}(86 \%)$ and aac $\left(6^{\prime}\right)$-Ii (86\%) were observed, followed by vanC (8\%), tetM (3\%). The Tn5801-tetM-like integrative conjugative element (ICE) was identified in E. gallinarum, exhibiting resistance to tetracycline $(64 \mu \mathrm{g} / \mathrm{mL})$. Seven probiotic E. faecalis and E. faecium, as active ingredients in human probiotics, shared the same STs (sequence types) and were distinct from the STs of other contaminated or mislabeled enterococci, indicating that two particular STs belonged to native probiotic isolates. These findings advocate appropriate assessments of enterococci when used in probiotics.

Keywords: probiotics; enterococci; hemolysis; virulence factors; biofilm; antimicrobial resistance; transposon ICE

\section{Introduction}

In the last few years, probiotics have been increasingly used in human, animal, aquaculture, and plant health worldwide. Probiotics are not only applied for healthcare, feed additive growth promotion and so on, but are also emerging as novel therapeutic tools for 
treating diseases such as FGIDs (functional gastrointestinal diseases) and septicemia [1,2]. The probiotic market in America is the most advanced, with China and Japan having occupied over half of the Asia-Pacific probiotic sales for decades.

A probiotic is a live microorganism isolated from different genera and species and is generally recognized as safe (GRAS) and effective. Among numerous microorganisms, lactic acid bacteria (LAB) and Bifidobacterium are the most popular and widely utilized as probiotic candidates worldwide. Enterococci, one kind of LAB, are commensal organisms that are well suited to survival in the gastrointestinal tract of human and animal and environments like water and soil. Enterococci have been identified as opportunistic pathogens that cause various infections, among which approximately $80 \%$ are associated with Enterococcus faecalis [3]. More importantly, the emergence and spread of vancomycinresistant Enterococcus (VRE) isolates presents serious therapeutic difficulty, owing to a lack of effective antimicrobial therapy [4]. In fact, quite a few enterococcal strains have been authorized as probiotics for use in pharmaceutical preparations or animal feed additives for decades, such as E. faecium Medilac-Vita (treatment of infantile enteritis, China), E. faecium SF68 ${ }^{\circledR}$ (dietary supplementation for human and animal, Switzerland), E. faecium Cylactin ${ }^{\circledR}$ (feed additive for animal, Switzerland), E. faecalis Symbioflor ${ }^{\circledR} 1$ (treatment of respiratory illness, Germany), and E. faecalis TH10 Dr. Ohhira ${ }^{\circledR}$ (dietary supplementation for human, Republic of Estonia). However, the FDA (Food and Drug Administration) of Taiwan, Province of China, has restricted the use of E. faecium and E. faecalis as food supplements in probiotics since July of 2018, owing to the widespread of multi-resistant enterococci, irrespective of origins and the isolated location. As Enterococcus has not yet been authenticated with the status of GRAS by the FDA, a limited number of enterococci as probiotics have been commercialized [5]. The mainly controversial issue of enterococci use in probiotics is the risk of their pathogenicity, i.e., virulence genes and antibiotic resistance genes (ARGs) of this genus being transferred horizontally to commensal gut microflora via the food chain, and the lack of legislation $[4,5]$.

In this study, we analyzed the contamination, label accuracy, and hemolysis of commercial probiotic products for human, animal, aquaculture, and plant use. The hemolytic activity, cytotoxicity, virulence factors, biofilm formation, antimicrobial resistance, and molecular types of enterococci originating from these various products are analyzed. These findings provide new scientific insights into the safety assessment of probiotic products and enterococcal isolates.

\section{Materials and Methods}

\subsection{Isolation and Identification of Enterococcus spp. from Probiotic Products}

The commercial probiotic products were collected between 2018-2020 (Supplementary Table S1). After the solid probiotic products were fully ground, $2 \mathrm{~g}$ of probiotic powder and $2 \mathrm{~mL}$ of the liquid probiotic product were suspended in $20 \mathrm{~mL}$ of sterile phosphate buffered saline (PBS). The probiotic suspensions were cultured in the $500 \mu \mathrm{L}$ brain heart infusion (BHI) broth containing $6.5 \% \mathrm{NaCl}$ and were incubated at $37^{\circ} \mathrm{C}$ for $8 \mathrm{~h}$ for preenrichment, and the inoculum size in the broth was $2 \%$. Afterwards, the bacterial cultures were spread on Enterococcus-selective media (Beijing Land Bridge Technology, Beijing, China) and incubated at $37^{\circ} \mathrm{C}$ for $18 \mathrm{~h}$. Brown single colonies of each distinct phenotype were sub-cultured in $1 \mathrm{~mL}$ BHI broth. Thereafter, the overnight cultures were diluted and spread-plated onto agar plates so that the isolated colonies could be obtained for further study.

Genomic DNAs were extracted from isolates using the TIANamp Genomic DNA Kit (Tiangen Biotech, Beijing, China), and the 16S rDNA gene was amplified using bacterial universal primers $27 \mathrm{~F}$ and $1492 \mathrm{R}$. The obtained sequences were analyzed using the nucleotide basic local alignment search tool (BLAST) [6] at NCBI for further identification of isolates from the probiotic products. 


\subsection{Toxin Production, Hemolysis Detection, and Cytotoxicity Assays}

Hemolysis tests and cytotoxicity assays were performed in order to assess the biological safety of both the probiotic products and their Enterococcus isolates. After suspending $2 \mathrm{~g}$ of probiotic powder and $2 \mathrm{~mL}$ of the liquid probiotic in $20 \mathrm{~mL}$ of PBS, suspensions were cultured in $10 \mathrm{~mL}$ lysogeny broth (LB) medium at $30^{\circ} \mathrm{C}$, and the inoculum size in the broth was $2 \%$. Meanwhile, Enterococcus isolates were cultured with an identical inoculum size in the broth. Both overnight supernatant cultures of probiotic products and isolates were harvested by centrifugation at $13,000 \mathrm{rpm}$ for $5 \mathrm{~min}$ and stored at $-20^{\circ} \mathrm{C}$ until used for toxin production analysis.

The hemolysis of each probiotic product and isolate was determined according to the method described previously with minor modification [7]. Briefly, sheep red blood cells (SBCs) (Hongquan Bio, Guangzhou, China) were harvested by centrifugation at $3000 \mathrm{rpm}$ for $10 \mathrm{~min}$, followed by rinsing twice with PBS. After 8 vol \% suspensions of SBCs were prepared using PBS, the suspensions were mixed with equal volumes of culture supernatants to generate final suspensions of $4 \%(\mathrm{vol} / \mathrm{vol}) \mathrm{SBCs}$, and then were incubated at $37^{\circ} \mathrm{C}$ for $1 \mathrm{~h}$, then finally harvested by centrifugation at $3000 \mathrm{rpm}$ for $10 \mathrm{~min}$ at $4{ }^{\circ} \mathrm{C}$. The $100-\mu \mathrm{L}$ concentrate sample in each tube was transferred to a sterile 96-well plate and the hemolytic activity was assessed by measuring the optical absorbance at $\mathrm{OD}_{576}$ with a microplate absorbance spectrophotometer (Bio-Rad, Hercules, CA, USA). Human red blood cells (RBCs) (Hongquan Bio, Guangzhou, China) were harvested by centrifugation for $3000 \mathrm{rpm}$ for $10 \mathrm{~min}$, followed by rinsing twice with PBS. After the suspensions of RBCs were prepared using PBS, the suspensions and molten LB agars $\left(50^{\circ} \mathrm{C}\right)$ were mixed in sterile culture dishes to generate final agars of $5 \%(\mathrm{vol} / \mathrm{vol}) \mathrm{RBCs}$. The overnight cultures of isolates were sub-cultured via streaking on RBC agar plates and were incubated at $37^{\circ} \mathrm{C}$ for $48 \mathrm{~h}$. Each plate was checked for single colony growth and assessment of the hemolytic zone.

The $10 \mu \mathrm{L}$ culture supernatants (for toxin production) of the isolates were used to test the cytotoxicity on Vero cells as previously described [8]. The water-soluble tetrazolium salt-8 (WST-8, MCE) method and a microscope were also used to determine whether the isolates were cytotoxic. Vero cells were obtained from Professor Kui Zhu at China Agricultural University. All tests were repeated three times and the significant difference was calculated.

\subsection{Antimicrobial Susceptibility Test}

The microdilution broth method, as described by the CLSI (Clinical Laboratory and Standards Institute) documents $[9,10]$, was used to determine the susceptibility of Enterococcus to 11 antimicrobial agents, including ampicillin, erythromycin, tetracycline, tigecycline, ciprofloxacin, gentamicin, streptomycin, linezolid, florfenicol, vancomycin, and teicoplanin. The MIC (minimum inhibitory concentration) ranges of the antimicrobial agents and the resistance breakpoints of the antimicrobial agents were found via referral to the CLSI documents.

\subsection{Whole-Genome Sequencing}

The whole genome of Enterococcus was sequenced on an Illumina NovaSeq 6000 platform with the $150 \mathrm{bp}$ paired-end module, and the sequencing library was generated using the VAHTS Universal DNA Library Prep Kit for Illumina ${ }^{\circledR}$ (Vazyme ND604, Nanjing, China) following manufacturer's recommendations. High-quality reads were de novo assembled using the SPAdes software v3.1.0 and annotated using the Prokka v1.12.0.

In order to screen isolates for the presence of virulence factors and ARGs, the core genome tree of Enterococcus was constructed using panX (pan-genome-analysis pipeline), and the whole genome of Enterococcus was analyzed using Blastn v2.11.0 (identity $\geq 80 \%$ and coverage $\geq 80 \%$ ) via the following databases, respectively: Virulence Factors of Pathogenic Bacteria (VFDB) (accessed on 10, Dec 2020) [11], the reported virulence factors [12], ResFinder v4.0 [13], and PointFinder v4.0 [14]. MLST (multilocus sequence typing) 
of the seven housekeeping genes ( $g d h, g y d, p s t S, g k i$, aroE, xpt, and yiqL) for Enterococcus was performed with Enterococcus MLST databases [15]. The allelic profiles and the sequence types were generated by using the BLAST with the Enterococcus sequences in the MLST database (https:/ / pubmlst.org/databases/ (accessed on 10 December 2020)).

\subsection{Biofilm Assay}

Crystal violet assay biofilm mass was quantified using the crystal violet assay as previously described [16]. E. faecalis JH2-2 was used as the quality control strain.

\subsection{Statistical Analysis}

GraphPad Prism version 8.3 was used for all statistical analysis. Hemolysis products were tested with the unpaired t-test. Statistical significance was determined and recorded as follows: $p<0.001\left(^{* *}\right), p<0.01\left(^{* *}\right), p<0.05\left({ }^{*}\right)$, or not statistically significant if $p>0.05$.

\section{Results and Discussion}

\subsection{Identification of Enterococci, Contamination, and Label Inaccuracy}

The products were collected from South Korea, Australia, America, The Netherlands, and 21 provinces/municipalities/autonomous regions (P/M/A) in China (Supplementary Table S1), covering more than half of the provincial administrative regions in China. In total, 110 probiotic products, including 47 human products used for preventive care and treatment, 34 animal products used for precaution, therapy, food additive, and excreta degradation, 14 aquaculture products used for water purification, and 15 plant products used for biocontrol and growth promotion, were examined. The active ingredients of all products are described in Supplementary Table S1. Based on the colony morphologies of selected media and 16S rDNA gene sequences from the whole genome sequence analysis, 36 Enterococcus spp. (31 E. faecium, 2 E. faecalis, 2 E. casseliflavus, and 1 E. gallinarum) were isolated and identified from 110 probiotic products (Figure 1 and Supplementary Table S1).

Among all 13 products labeled with ingredients containing Enterococcus spp., no Enterococcus was isolated from five animal products (No. 61, 63, 72, 78, and 79), while one E. faecium isolate and one E. gallinarum isolate were identified in two animal products (No. 65 and 74) labeled with ingredients containing E. faecalis. Over half of the products labeled with ingredients containing Enterococcus spp. were below the standard. This result is in accordance with previous findings $[17,18]$ where the active ingredient was missing in quite a few probiotic products.

Furthermore, 26 E. faecium and 2 E. casseliflavus isolates were identified in 27 products (from all four origins) labeled with ingredients not containing Enterococcus, suggesting that these products were contaminated by these two species. Compared with E. faecalis, E. faecium has a high frequency of multi-resistant phenotypes and composes the majority of VRE infections [19]. Interestingly, E. casseliflavus and E. gallinarum, which are not authorized for use in probiotics, were present in two probiotic products. Besides, the overwhelmingly predominant species among enterococci, originating from diverse sources, are E. faecalis and E. faecium $[20,21]$. Therefore, it is unknown how these probiotic products were contaminated by E. casseliflavus and E. gallinarum. Since enterococci are widespread in the intestinal tracts of mammals and birds, and also in water and soil [20], this could explain the possibility of cross contamination by different Enterococcus sp. that are not listed as an ingredient in the probiotics considered here. Usually, the isolation rates of E. faecalis are higher than those of E. faecium, irrespective of the sample origin [4,20], while E. faecium, with an isolation rate of $86 \%$, was the predominant species in this study. Inappropriately, quite a few probiotic products are directly labeled with ingredients containing LAB, thereby causing confusion, since the term "LAB" includes several genera, such as Lactobacillus, Streptococcus, Pediococcus, and Enterococcus. Label inaccuracy for products from all four origins was also observed. 


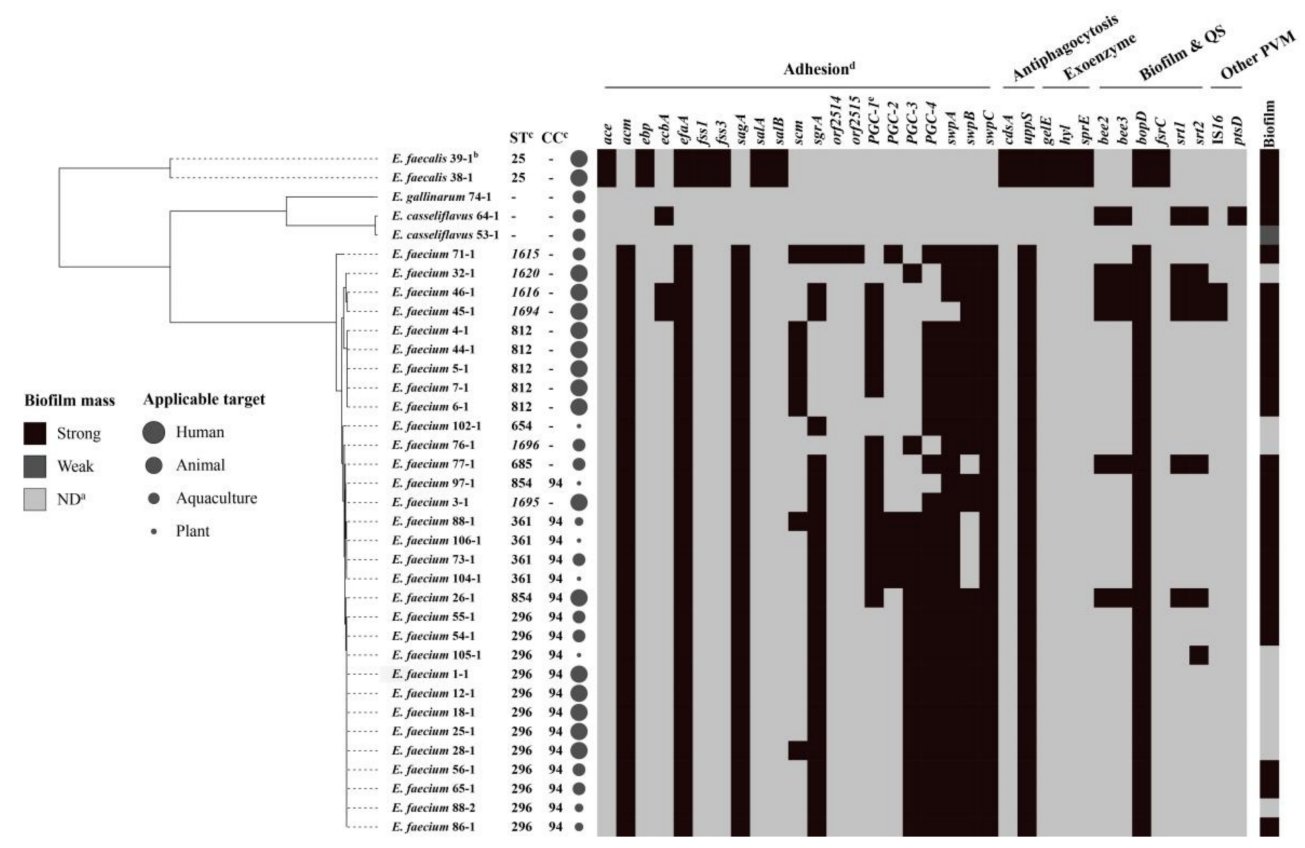

Figure 1. Virulence genes, MLST (multilocus sequence typing), and biofilm mass profiles of 36 Enterococcus spp. isolates from the probiotics. Note: (a) ND, no biofilm mass; PVM, putative virulence marker. (b) The rule of strain name is the first number and represents the originated probiotic product, and the latter number represents the isolated strain. (c) CC, clonal complex; the new STs (sequence types) were labelled in italic; -, ST or clonal complex cannot be assigned. (d) In the heatmap, black color indicates strain carrying the corresponding virulence gene. (e) PGC-1, a complete PGC-1 cluster, including pilA, pilE, pilF, orf1903, orf1905, and orf1916; PGC-2, a complete PGC-2 cluster, including orf2008, orf2009, and orf2010; PGC-3, a complete PGC-3 cluster, including pilB, pilE, pilF, orf2568, $\operatorname{orf} 2570$, and $\operatorname{orf} 2571$.

Together, these findings are in accordance with previous studies $[17,18,22]$ and confirm that the cross contamination, label inaccuracy, and lack of active ingredients in probiotic products, especially those for human use, could pose great risks to the health of humans and livestock.

\subsection{Hemolytic Activity and Cytotoxicity}

As depicted in Figure 2, among 110 probiotic products, 35 (31.8\%) showed hemolysis, including 5 from human products $(4.6 \%), 14$ from animal products $(12.7 \%), 8$ from aquaculture products $(7.3 \%)$, and 8 from plant products $(7.3 \%)$. In detail, 5 of 47 human products $(10.6 \%)$ exhibited hemolysis, with a maximum hemolysis rate of $93 \%$ for product No. 28 . Fourteen of 34 animal products ( $41.2 \%$ ) exhibited hemolysis, with a maximum hemolysis rate of $95 \%$ for product No. 48 , while over one half of the aquaculture and animal products exhibited hemolysis. The human products seem to be relatively secure, showing significant differences $(p<0.05)$ in hemolysis when compared with products of the other three origins.

Although most of hemolytic isolates belong to E. faecalis and E. faecium, the leading species in enterococcal infections, our results showed that no hemolysis or cytotoxicity was observed for 36 Enterococcus spp., indicating that these Enterococcus isolates were not responsible for the hemolysis activity of the products. There are different kinds of bacteria and compounds that can cause hemolysis, such as Bacillus, which is used in probiotics for humans and animals, showing cytotoxicity owing to Bacillus carrying the heat-labile enterotoxins $\mathrm{Nhe}$ and $\mathrm{Hbl}[22,23]$. To rule out the possibility of enterococci belonging to the LAB group (also those that like to produce acid) producing hemolytic toxins, both the probiotic products and enterococci were cultured in LB media instead of lactobacilli MRS broths, and the $\mathrm{pH}$ values of each culture were confirmed overnight. All cultures with a final $\mathrm{pH}$ between 6.0-8.0 were used for hemolysis detection. As a 
result, the hemolysis activity was caused by the products themselves, such as exotoxins secreted by culturable bacteria or/and endotoxins released when bacteria were ruptured or disintegrated. Nevertheless, the other ingredients in the product, or even contaminants existing in the product, could contribute to the hemolysis as cryptic virulence factors. As shown in Supplementary Table S1, over half of the products considered here contained more than one bacterial species and the frequency of hemolysis was $60.0 \%$ in products with genera of more than one class, and this was higher than those with a single genus. Moreover, microflora in probiotic bacteria cultures are likely to play a synergistic role in toxic substances. Based on these observations, it seems difficult to assess what potential hemolytic factors might exist for probiotic products. Whole-process supervision is the most important and effective measure to ensure the safety of probiotics.

\begin{tabular}{ccccc}
\hline & Num & Hemolytic & Nonhemolytic & $\begin{array}{c}\text { Hemolytic } \\
\text { Propotion (\%) }\end{array}$ \\
\hline Human & 47 & 5 & 42 & $11 \%$ \\
Animal & 34 & 14 & 20 & $41 \%$ \\
Aquaculture & 14 & 8 & 6 & $57 \%$ \\
Plant & 15 & 8 & 7 & $53 \%$ \\
Total & 110 & 35 & 75 & $32 \%$ \\
\hline
\end{tabular}

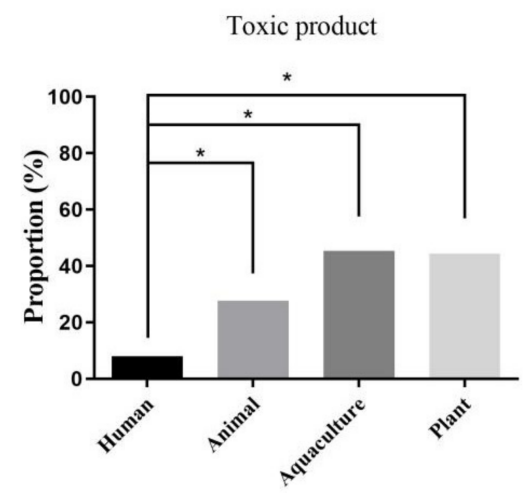

Figure 2. The proportion of toxic products in 110 probiotic products from four origins. Among 110 probiotic products, $35(31.8 \%)$ showed hemolysis, including 5 from human products $(4.6 \%)$, 14 from animal products $(12.7 \%), 8$ from aquaculture products $(7.3 \%)$, and 8 from plant products (7.3\%). Simultaneously, 5 of 47 human products (10.6\%) exhibited hemolysis. In total, 14 of 34 animal products $(41.2 \%)$ exhibited hemolysis, while over one half of aquaculture and animal products exhibited hemolysis. The human products showed significant difference $\left.{ }^{*} p<0.05\right)$ in the hemolysis compared with the products of the other three origins.

\subsection{Virulence Factors Associated with Adhesion, Antiphagocytosis, Exoenzymes, and Biofilm Mass}

Although no toxic phenotypes of the isolates were observed in terms of hemolysis and cytotoxicity, the virulence genotypes of 36 Enterococcus spp. were determined. A brief introduction of virulence factor functions is described in Supplementary Table S2. Except for the factors associated with exoenzymes only detected in two probiotic E. faecalis from human products $(5.6 \%)$, the virulence factors associated with adhesion $(94.4 \%)$, antiphagocytosis (91.7\%), biofilm formation (94.4\%), and other PVM (8.3\%) were extensively identified in enterococci isolated from human, animal, aquaculture, and plant probiotics (Figure 3). The prevalence rates of factors associated with adhesion, antiphagocytosis, and biofilm formation in enterococci from human, aquaculture, and plant probiotics were $47.2 \%, 8.3 \%$, and $13.9 \%$, respectively, while these three factors in enterococci from animal probiotics were $25 \%, 22.2 \%$, and $25 \%$, respectively. Interestingly, all enterococci isolates did not cause hemolysis or cytotoxicity. It could be explained because no exotoxin factor, such as cytolysin, resulting in lysing erythrocytes and gram-positive bacteria, was observed in 36 Enterococcus spp. 


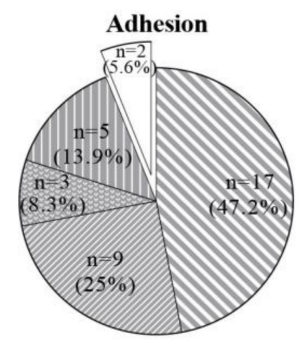

Human
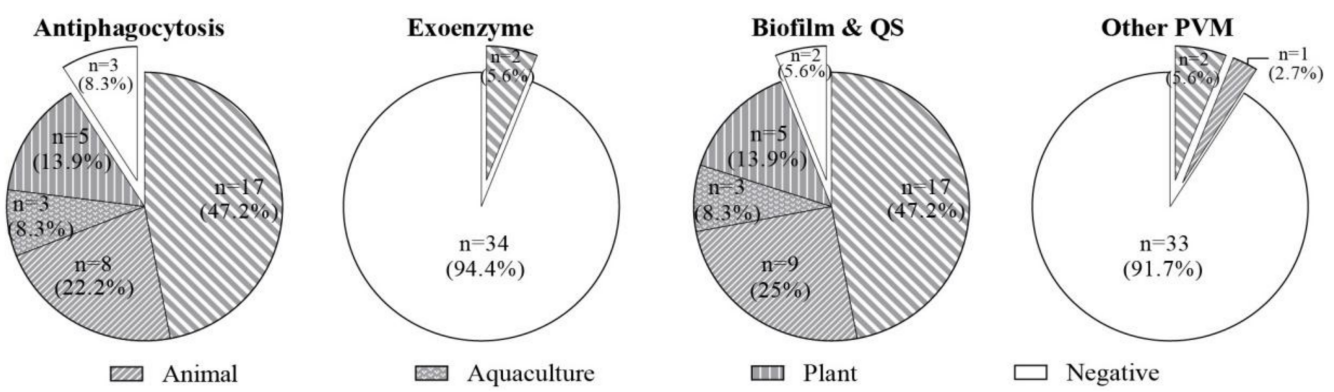

Figure 3. The virulence profile of 36 Enterococcus spp. isolates from four origins. The detection rates of virulence factors associated with adhesion, antiphagocytosis, exoenzyme, biofilm, and other PVM in 36 Enterococcus spp. were 94.4\%, 91.7\%, $5.6 \%, 94.4 \%$, and $8.3 \%$. In the above diagrams, negative indicates an isolate not carrying the corresponding virulence gene. Except for factors associated with exoenzymes merely detected in two probiotic E. faecalis from human products $(5.6 \%)$, prevalence rates of factors associated with adhesion, antiphagocytosis, and biofilm in enterococci from human, aquaculture, and plant probiotics were $47.2 \%, 8.3 \%$, and $13.9 \%$, respectively, while these three factors in enterococci from animal probiotics were $25 \%, 22.2 \%$, and $25 \%$.

After screening each isolate for the presence of major virulence genes, the results showed that efaA, BopD, and uppS were all detected in E. faecium and E. faecalis (Figure 1). This was evidenced by the widespread occurrence of these virulence factor-associated proteins in enterococci, whereas 28 virulence factors were rarely detected in E. casseliflavus and E. gallinarum. Similarly, numerous virulence factors in the genus were mainly found in species of E. faecalis and E. faecium isolates from diverse sources, except from probiotic products [24]. It seems that $a c e, e b p, f s s, s a l A, s a l B, c d s A, f s r C$, and three exoenzyme factors were restricted to $E$. faecalis here. In all E. faecalis, genes of gelatinase (GelE), serine protease (SprE), and hyaluronidase were active, so these may contribute to host tissue invasion. Because hyaluronidase produced by enterococci isolated from patients with abscesses has been described as a spreading factor for worms [25], this supports the importance of this exoenzyme in the infection process. Especially, the probiotic isolates E. faecalis 38-1 and 39-1 were indicated as the active ingredients in human probiotic products No. 38 and 39 (Supplementary Table S1), rather than contaminants, and this represents a significant threat to the health of food and humans because of this positive genotype.

Genes associated with biofilm formation and quorum sensing (QS) were found in 34 Enterococcus spp. (94.4\%). The Gene bopD, involved in biofilm production, was widespread in E. faecium. Gene encoding sortase (Srt), which is important for the biofilm production of E. faecalis, has been isolated from a patient with endocarditis [26] and has been found in 19\% of enterococci. Simultaneously, gene encoding endocarditis and biofilm-associated pili (Ebp pili, ebp), which are not only important for adherence to host extracellular matrix proteins (including fibrinogen and collagen), but also play an important role in endovascular infection [26], were also found in probiotic E. faecalis of human probiotic products No. 38 and 39. By contrast, the bee gene (biofilm enhancer in Enterococcus), which has been confirmed to confer a high biofilm-forming phenotype to E. faecalis [27], was found in five isolates of E. faecium and one isolate of E. casseliflavus here.

The biofilm formation of enterococci represents a critical safety issue in both the healthcare field and the food industry, since E. faecalis isolated from endocarditis produces biofilms significantly more often than nonendocarditis Enterococcus [28]. In total, 26 of 36 enterococci $(72.2 \%)$ exhibited an ability of biofilm formation, where $69.4 \%, 0 \%$, and $2.8 \%$ of them were strong, moderate, and weak biofilm producers (Table 1). The enterococci isolated from animal probiotics showed a significantly higher frequency of strong biofilm formation when compared with enterococci isolated from the other three origins. Notably, among 36 enterococci, two E. faecalis (38-1 and 39-1) and four E. faecium (4-1, 5-1, 6-1, and 7-1) strongly formed biofilms and were all listed as active ingredients in human probiotics. The widespread presence of the biofilm phenotype in enterococci, irrespective of origin, suggests interspecies transmission of biofilm-associated genes. 
Table 1. The strength of biofilm formation among 36 Enterococcus spp.

\begin{tabular}{ccccc}
\hline Origin & Strong Biofilm & $\begin{array}{c}\text { Moderate } \\
\text { Biofilm }\end{array}$ & Weak Biofilm & Total \\
\hline Human & $11 / 17$ & 0 & 0 & $11 / 17$ \\
Animal & $9 / 11$ & 0 & $1 / 11$ & $10 / 11$ \\
Aquaculture & $2 / 3$ & 0 & 0 & $2 / 3$ \\
Plant & $3 / 5$ & 0 & 0 & $3 / 5$ \\
Total & $25 / 36$ & 0 & $1 / 36$ & $26 / 36$ \\
\hline
\end{tabular}

The food chain has been considered as an important source for the dissemination of enterococci to humans and animals. In many cases, the oral administration of probiotic Enterococcus carrying potential virulence factors for patients with immunodeficiency syndrome, diarrhea, or IBD (inflammatory bowel disease) may cause significant infections and diseases, such as endocarditis and bacteremia [4]. Based on these observations, we speculate that the production of different virulence factors may be beneficial for the survival of Enterococcus spp. in diverse environments and is likely to contribute to enterococci being the predominant contaminants during production processes for probiotic products. For a Qualified Presumption of Safety (QPS) status, the EFSA (European Food Safety Authority) requires demonstration of the absence of virulence factors of probiotic strains. Adhesion, hemolysin, hyaluronidase, biofilms and so on are putative virulence factors which should be included in Enterococcus because they are likely to be transferred to the gut microflora of humans and animals via the oral administration of probiotics, and eventually contributing to the environmental reservoir of virulence factors.

\subsection{Antimicrobial Resistance and Genetic Environment of tetM}

The MIC breakpoints for ampicillin, erythromycin, tetracycline, tigecycline, ciprofloxacin, linezolid, florfenicol, vancomycin, and teicoplanin are 16, 8, 16, 0.25, 4, 8, 32, 32, and $32 \mu \mathrm{g} / \mathrm{mL}$, respectively. As shown in Figure 4 and Supplementary Table S3, 35 of 36 Enterococcus spp. (97\%) were resistant to erythromycin, which is in accordance with the results of $86 \%$ enterococci either carrying $m s r C$ or $l s a A$, which is intrinsic to E. faecalis and confers low-level macrolide resistance $[29,30]$. No ARG associated with macrolides was observed in E. casseliflavus 64-1 and 53-1, indicating that a novel determinant could play a part in the erythromycin phenotype in 64-1 and 53-1. Notably, most infective E. faecium isolates are ampicillin resistance with an MIC of ampicillin of $>16 \mu \mathrm{g} / \mathrm{mL}$ [31]. On the other hand, a strain that has an MIC of ampicillin of $\leq 2 \mu \mathrm{g} / \mathrm{mL}$ and lacks all IS16, esp and hyl genes should be regarded as safe [12]. Overall, 27 of 36 enterococci (75\%) had an MIC of ampicillin of $>2 \mu \mathrm{g} / \mathrm{mL}$, and 8 E. faecium were found to have the mutations of $p b p 5$ conferring ampicillin resistance, which may be a potential risk factor associated with the prevalence and distribution of ARGs of probiotic enterococci.

The result that only a few isolates exhibited low-level resistance to vancomycin (8\%) and ciprofloxacin $(8 \%)$ is opposite to the majority of findings that have suggested that $E$. faecalis and E. faecium originated from the other sources, such as animals, water, and soil, have multidrug resistance phenotypes and high-level antimicrobial resistance. Among six known genes (van $A, \operatorname{van} B, \operatorname{van} C, \operatorname{van} D$, van $E$, and $v a n G)$ of glycopeptide resistance in Enterococcus spp., vanA is the most important and prevalent enterococcal ARG and confers high-level vancomycin and teicoplanin resistance to E. faecium and E. faecalis (namely VRE) [4]. No Enterococcus was positive for vanA, whereas vanC, conferring low-level vancomycin resistance [32], was detected in the less commonly encountered E. casseliflavus and E. gallinarum strains (Supplementary Table S3). Unexpectedly, 33 (2 E. faecalis and 31 E. faecium) of 36 enterococci were negative for $v a n C$, which is an intrinsic trait of $E$. casseliflavus and E. gallinarum [33]. Generally, both E. gallinarum and E. casseliflavus are less pathogenic and show lower antimicrobial resistance than E. faecium and E. faecalis, but E. gallinarum and E. casseliflavus carrying vanC may influence host immunity, as suggested in recent reports [32]. No ARG conferring ciprofloxacin resistance was observed in 36 
Enterococcus spp., suggesting that the ciprofloxacin resistance found in three enterococci may be associated with mutations in the quinolone resistance-determining region (QRDR) of the gyrA gene.

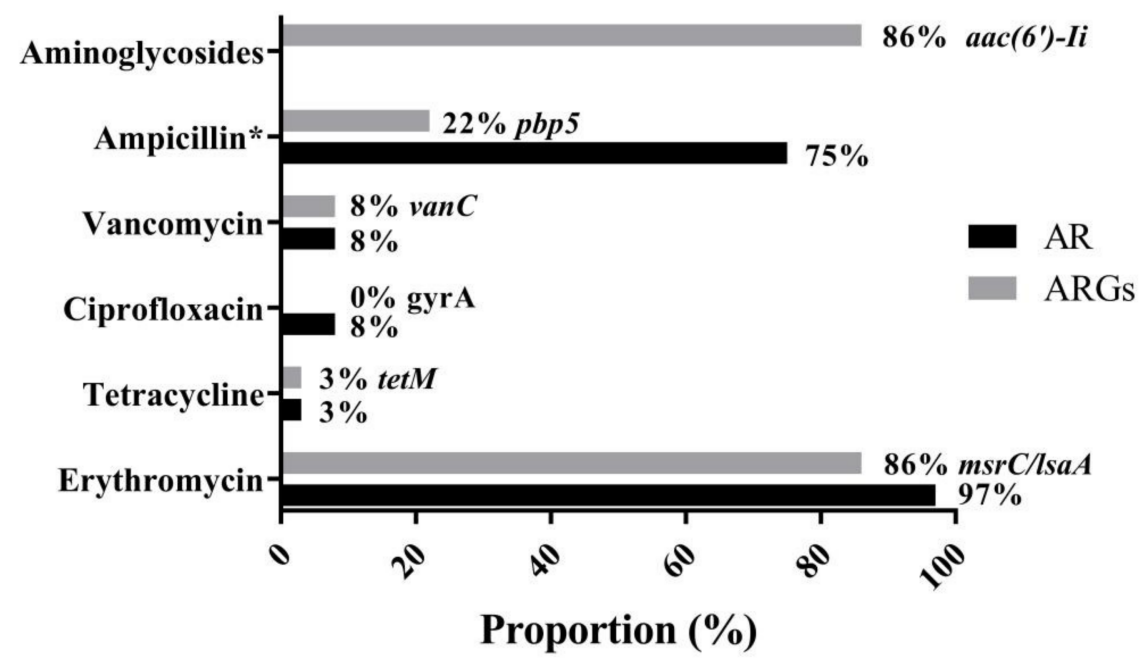

Figure 4. Major antimicrobial resistance phenotype (AR, antibiotic resistance) and genotype (ARGs, antibiotic resistance genes) in 36 enterococci. Resistance rates against erythromycin (97\%), vancomycin and ciprofloxacin ( $8 \%$ ), tetracycline $(3 \%)$, and aminoglycosides $(0 \%)$ were found in 36 enterococci, respectively. ${ }^{*}$ indicates an isolate with an MIC of ampicillin of $>2 \mu \mathrm{g} / \mathrm{mL}$. High detection rates of $m s r C / l s a A(86 \%)$, aac $\left(6^{\prime}\right)$-Ii (86\%), followed by pbp5 (22\%), vanC (8\%), tetM (3\%), and ciprofloxacin resistance gene $(0 \%)$ were observed, respectively.

Intrinsic antimicrobial resistance of enterococci includes aminoglycosides [33], and no isolate with high-level gentamicin resistance (HLGR) or high-level streptomycin resistance (HLSR) was presented in this study. Generally, the frequency of HLGR of isolates that exhibited high rates of multiple antibiotic resistance was higher than HLSR in enterococci [34,35], and both HLGR and HLSR were much more frequently detected in VRE [36]. However, 30 of 31 E. faecium were positive to aac $\left(6^{\prime}\right)$-Ii, which is intrinsic and specific for $E$. faecium and encodes an aminoglycoside $6^{\prime}-\mathrm{N}$-acetyltransferase, conferring medium levels of most aminoglycosides [37].

Similarly, as reported in many studies [4], both the genotypes and phenotypes associated with erythromycin, vancomycin, and aminoglycoside resistance are intrinsic to enterococci. In contrast, only one Enterococcus, E. gallinarum 74-1, from an animal probiotic product, showed an intermediate level in the MIC $(64 \mu \mathrm{g} / \mathrm{mL})$ of tetracycline, and tet $M$ was involved in acquired resistance of Enterococcus to tetracycline (Figure 4, Supplementary Table S3). The genetic environment of tet $M$ in E. gallinarum 74-1 was further determined. The sequence of a chromosomal element of 24,265 bp containing the tet $M$ gene and site-specific integrase was inserted between the gene encoding LPXTG cell wall anchor domain-containing protein (accession no. WP_142972242) and guaA, encoding glutamine-hydrolyzing GMP synthase, and the G + C content of this element was 35\% (Figure 5), which is significantly lower than that $(40.5 \%)$ of the entire genome of E. gallinarum ASM214049v1. This unique element, having a central region and carrying the gene tet $M$, the only ARG in the element, exhibited an overall nucleotide sequence identity of $99.9 \%$ to the tetM transposon of E. avium FDAARGOS184 (accession no. CP024590), E. faecalis CVM N60443F (accession no. CP028724), and S. aureus NF25 (accession no. CP035415) [38], and thus belonged to the Tn5801-like ICE (integrative conjugative element). Interestingly, Tn5801 of E. faecium E240 (accession no. KP001176) integrated into tetM, resulting in tet $M$ being truncated and showing no tetracycline phenotype (Figure 5). Tn5801, originally detected in Staphylococcus aureus, was a Tn916 family element in which the genes int and xis in Tn916 were replaced by a unique int gene (int5801) [39]. Importantly, a similar 
transposon ICE was also observed in VRE isolates (Figure 5) [38]. Enterococcus, particularly VRE, has the ability to transfer ARGs to produce frequent virulence characteristics, such as hemolysin, hyaluronidase, and adhesion [40].

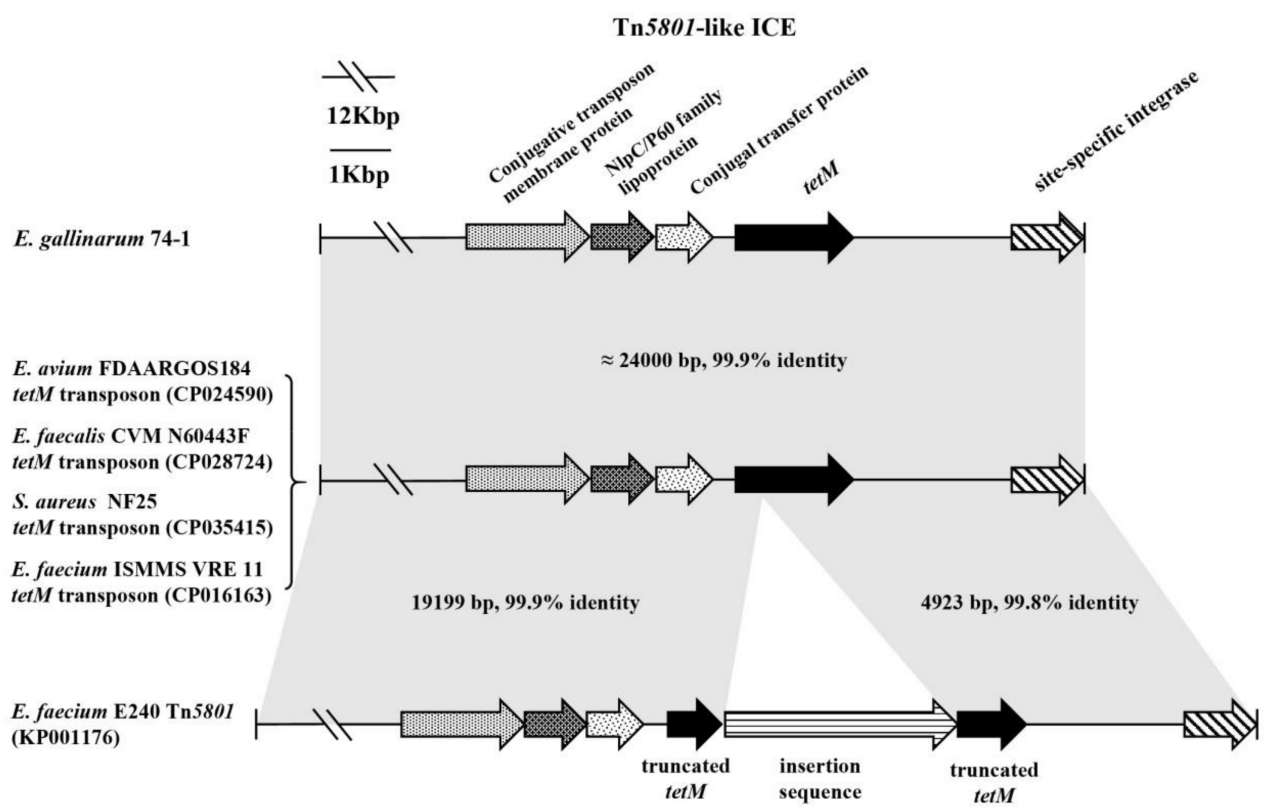

Figure 5. Tn 5801-like ICE in E. gallinarum 74-1 compared with selected transposon ICEs (integrative conjugative elements) from VRE (vancomycin-resistant Enterococcus), other enterococci, and S. aureus. The gray shading indicates regions sharing more than $99 \%$ DNA identity. The tetM gene was truncated into two parts in Tn5801 of E. faecium E240.

In short, some enterococci of different origins may be from very diverse genetic lineages and environments, but the genus is capable of rapidly acquiring ARGs and mobile genetic elements, resulting in the dissemination of antibiotic resistance (AR) and ARGs among isolates, irrespective of their source or location.

\subsection{Molecular Typing among Probiotic Isolates and Contaminated or Mislabeled Isolates}

Among 33 E. faecalis and E. faecium, MLST yielded 13 sequence types (STs) (Figure 1), four of which, ST1615, ST1620, ST1616, and ST1694, were newly assigned in this study. No E. casseliflavus and E. gallinarum data were available according to the MLST database. All 13 STs, except for 15 singletons, were clustered into one clonal complex: CC94. Notably, all seven probiotic E. faecalis and E. faecium as active ingredients in human probiotic products shared the same STs (25 and 812, respectively), which were distinct from the STs of the other contaminated or mislabeled enterococci in probiotic products, indicating that these two particular sequence types belong to native probiotic isolates. MLST analysis of probiotic strains and isolates from probiotic products could thus be recommended to provide some indication as to whether the strain or isolate belongs to a problematic lineage.

\section{Conclusions}

This study provides comprehensive evidence for the hemolytic activity, label inaccuracy, high level of contamination of E. faecium, and the lack of active ingredients in probiotic products for human, animal, aquaculture, and plant use. Our findings also showed that virulence traits, a strong biofilm formation ability, and ARGs, particularly those carrying a transposon ICE, can be found in probiotic E. faecalis and E. faecium and other contaminated enterococci, irrespective of their origin, suggesting that such virulence factors and antimicrobial resistance determinants may transfer into the gastrointestinal system in humans and animals. Thus, in future worldwide applications of probiotics, our findings suggest that the probiotic potential of enterococci requires appropriate assessment to exclude the presence 
of virulence factors, antimicrobial resistance traits, and gene transfer risk, especially in countries where enterococci are commercialized as probiotic products.

Supplementary Materials: The following are available online at https:/ / www.mdpi.com/article/10 .3390 / microorganisms 9040726 / s1, Table S1: Information of commercial probiotic products, Table S2: Brief introduction of virulence factor function, Table S3: Genotype and phenotype of antimicrobial resistance in 36 Enterococcus spp. Isolates.

Author Contributions: F.D.: Conceptualization, Project administration, Writing一original draft \& review \& editing, Funding acquisition. Y.C.: Conceptualization, Methodology, Validation, Formal analysis, Investigation. X.Z.: Methodology, Validation, Formal analysis. H.X. and T.S.: Investigation. Y.D.: Conceptualization, Project administration, Methodology, Supervision, Validation. J.W.: Conceptualization, Methodology, Writing-review \& editing, Funding acquisition. All authors have read and agreed to the published version of the manuscript.

Funding: This work was funded by grants from the National Key Research and Development Program of China (No. 2017YFC1600305), the National Natural Science Foundation of China (No. 31802246), and the Science and Technology Program of Guangzhou (No. 201804010207).

Institutional Review Board Statement: Not applicable.

Informed Consent Statement: Not applicable.

Data Availability Statement: The draft whole genome sequence assemblies of the 36 Enterococcus spp. have been deposited in GenBank under BioProject PRJNA703331 https:/ / www.ncbi.nlm.nih. gov/bioproject/PRJNA703331 (accessed on 3 March 2021).

Acknowledgments: We acknowledge Kui Zhu (China Agricultural University) for providing Vero cells.

Conflicts of Interest: The authors declare no conflict of interest.

\section{References}

1. Giannetti, E.; Staiano, A. Probiotics for irriFIG bowel syndrome: Clinical data in children. J. Pediatr. Gastroenterol. Nutr. 2016, 63 (Suppl. 1), S25-S26.

2. Jenke, A.; Ruf, E.-M.; Hoppe, T.; Heldmann, M.; Wirth, S. Bifidobacterium septicaemia in an extremely low-birthweight infant under probiotic therapy: Figure 1. Arch. Dis. Child. Fetal Neonatal Ed. 2012, 97, F217-F218. [CrossRef]

3. Werner, G.; Coque, T.M.; Hammerum, A.M.; Hope, R.; Hryniewicz, W.; Johnson, A.; Klare, I.; Kristinsson, K.G.; Leclercq, R.; Lester, C.H.; et al. Emergence and spread of vancomycin resistance among enterococci in Europe. Eurosurveillance 2008, $13,19046$. [CrossRef]

4. Ben Braiek, O.; Smaoui, S. Enterococci: Between Emerging Pathogens and Potential Probiotics. BioMed Res. Int. 2019, 2019, 1-13. [CrossRef]

5. Franz, C.M.A.P.; Huch, M.; Abriouel, H.; Holzapfel, W.; Gálvez, A. Enterococci as probiotics and their implications in food safety. Int. J. Food Microbiol. 2011, 151, 125-140. [CrossRef] [PubMed]

6. Johnson, M.; Zaretskaya, I.; Raytselis, Y.; Merezhuk, Y.; McGinnis, S.; Madden, T.L. NCBI BLAST: A better web inter-face. Nucleic. Acids Res. 2008, 36, 5-9. [CrossRef] [PubMed]

7. Liu, Y.; Ding, S.; Dietrich, R.; Märtlbauer, E.; Zhu, K. A Biosurfactant-Inspired Heptapeptide with Improved Specificity to Kill MRSA. Angew. Chem. Int. Ed. 2017, 56, 1486-1490. [CrossRef] [PubMed]

8. Zhu, K.; Acaröz, U.; Märtlbauer, E. A cellular logic circuit for the detection of bacterial pore-forming toxins. Chem. Commun. 2013, 49, 5198. [CrossRef]

9. Clinical and Laboratory Standards Institute (CLSI). Performance Standards for Antimicrobial Disk and Dilution Susceptibility Tests for Bacteria Isolated from Animals. Third Edition: Approved Standard M31-A3; Clinical and Laboratory Standards Institute: Wayne, PA, USA, 2008.

10. Clinical and Laboratory Standards Institute (CLSI). Performance Standards for Antimicrobial Susceptibility Testing; Twenty-Eight Informational Supplement M100-S28; Clinical and Laboratory Standards Institute: Wayne, PA, USA, 2019.

11. Liu, B.; Zheng, D.; Jin, Q.; Chen, L.; Yang, J. VFDB 2019: A comparative pathogenomic platform with an interactive web interface. Nucleic Acids Res. 2019, 47, D687-D692. [CrossRef]

12. Freitas, A.R.; Tedim, A.P.; Novais, C.; Coque, T.M.; Peixe, L. Distribution of putative virulence markers in Entero-coccus faecium: Towards a safety profile review. J. Antimicrob. Chemother. 2018, 73, 306-319. [CrossRef] [PubMed]

13. Bortolaia, V.; Kaas, R.S.; Ruppe, E.; Roberts, M.C.; Schwarz, S.; Cattoir, V.; Philippon, A.; Allesoe, R.L.; Rebelo, A.R.; Florensa, A.F.; et al. ResFinder 4.0 for predictions of phenotypes from genotypes. J. Antimicrob. Chemother. 2020, 75, 3491-3500. [CrossRef] 
14. Zankari, E.; Allesøe, R.; Joensen, K.G.; Cavaco, L.M.; Lund, O.; Aarestrup, F.M. PointFinder: A novel web tool for WGS-based detection of antimicrobial resistance associated with chromosomal point mutations in bacterial pathogens. J. Antimicrob. Chemother. 2017, 72, 2764-2768. [CrossRef]

15. Jolley, K.A.; Bray, J.E.; Maiden, M.C.J. Open-access bacterial population genomics: BIGSdb software, the PubMLST.org website and their applications. Wellcome Open Res. 2018, 3, 124. [CrossRef]

16. Zheng, J.X.; Bai, B.; Lin, Z.W.; Pu, Z.Y.; Yao, W.M.; Chen, Z.; Li, D.Y.; Deng, X.B.; Deng, Q.W.; Yu, Z.J. Characterization of biofilm formation by Enterococcus faecalis isolates derived from urinary tract infections in China. J. Med. Microbiol. 2018, 67, 60-67. [CrossRef]

17. Cui, Y.; Wang, S.; Ding, S.; Shen, J.; Zhu, K. Toxins and mobile antimicrobial resistance genes in Bacillus probiotics constitute a potential risk for One Health. J. Hazard. Mater. 2020, 382, 121266. [CrossRef]

18. Weese, J.S.; Martin, H. Assessment of commercial probiotic bacterial contents and label accuracy. Can. Vet. J. Rev. Vet. Can. 2011, $52,43-46$.

19. Li, F.; Wang, Y.; Sun, L.; Wang, X. Vancomycin-resistant Enterococcus faecium pneumonia in a uremic patient on hemodialysis: A case report and review of the literature. BMC Infect. Dis. 2020, 20, 167. [CrossRef]

20. Byappanahalli, M.N.; Nevers, M.B.; Korajkic, A.; Staley, Z.R.; Harwood, V.J. Enterococci in the Environment. Microbiol. Mol. Biol. Rev. 2012, 76, 685-706. [CrossRef]

21. Harwood, V.J.; Delahoya, N.C.; Ulrich, R.M.; Kramer, M.F.; Whitlock, J.E.; Garey, J.R.; Lim, D.V. Molecular confirmation of Enterococcus faecalis and E. faecium from clinical, faecal and environmental sources. Lett. Appl. Micro-Biol. 2004, 38, 476-482. [CrossRef] [PubMed]

22. Zhu, K.; Hölzel, C.S.; Cui, Y.; Mayer, R.; Wang, Y.; Dietrich, R.; Didier, A.; Bassitta, R.; Märtlbauer, E.; Ding, S. Probiotic Bacillus cereus strains, a potential risk for public health in China. Front. Microbiol. 2016, 7, 718. [CrossRef]

23. Deng, F.; Chen, Y.; Sun, T.; Wu, Y.; Su, Y.; Liu, C.; Zhou, J.; Deng, Y.; Wen, J. Antimicrobial resistance, virulence characteristics and genotypes of Bacillus spp. from probiotic products of diverse origins. Food Res. Int. 2021, 139, 109949. [CrossRef] [PubMed]

24. Semedo, T.; Santos, M.A.; Lopes, M.F.; Marques, J.J.; Crespo, M.T.; Tenreiro, R. Virulence Factors in Food, Clinical and Reference Enterococci: A Common Trait in the Genus? Syst. Appl. Microbiol. 2003, 26, 13-22. [CrossRef] [PubMed]

25. Jett, B.D.; Huycke, M.M.; Gilmore, M.S. Virulence of enterococci. Clin. Microbiol. Rev. 1994, 7, 462-478. [CrossRef] [PubMed]

26. Nallapareddy, S.R.; Singh, K.V.; Sillanpää, J.; Garsin, D.A.; Höök, M.; Erlandsen, S.L.; Murray, B.E. Endocarditis and biofilmassociated pili of Enterococcus faecalis. J. Clin. Investig. 2006, 116, 2799-2807. [CrossRef]

27. Tendolkar, P.M.; Baghdayan, A.S.; Shankar, N. Putative Surface Proteins Encoded within a Novel Transferable Locus Confer a High-Biofilm Phenotype to Enterococcus faecalis. J. Bacteriol. 2006, 188, 2063-2072. [CrossRef]

28. Mohamed, J.A.; Huang, W.; Nallapareddy, S.R.; Teng, F.; Murray, B.E. Influence of Origin of Isolates, Especially Endocarditis Isolates, and Various Genes on Biofilm Formation by Enterococcus faecalis. Infect. Immun. 2004, 72, 3658-3663. [CrossRef]

29. Reynolds, E.; Cove, J.H. Enhanced resistance to erythromycin is conferred by the enterococcal msrC determinant in Staphylococcus aureus. J. Antimicrob. Chemother. 2005, 55, 260-264. [CrossRef]

30. Singh, K.V.; Weinstock, G.M.; Murray, B.E. An Enterococcus faecalis ABC homologue (Lsa) is required for the resistance of this species to clindamycin and quinupristindalfopristin. Antimicrob. Agents Chemother. 2002, 46, 1845-1850. [CrossRef]

31. De Regt, M.J.; van Schaik, W.; van Luit-Asbroek, M.; Dekker, H.A.; van Duijkeren, E.; Koning, C.J.; Bonten, M.J.; Willems, R.J. Hospital and community ampicillin-resistant Enterococcus faecium are evolutionarily closely linked but have diversified through niche adaptation. PLoS ONE 2012, 7, ee30319. [CrossRef]

32. Rashidi, A.; Zhu, Z.; Kaiser, T.; Manias, D.A.; Holtan, S.G.; Rehman, T.U.; Weisdorf, D.J.; Khoruts, A.; Dunny, G.M.; Staley, C. Vancomycin-resistance gene cluster, vanC, in the gut microbiome of acute leukemia patients undergoing in-tensive chemotherapy. PLoS ONE 2019, 14, e223890. [CrossRef]

33. Araújo, T.F.; Ferreira, C.L.D.L.F. The genus Enterococcus as probiotic: Safety concerns. Braz. Arch. Biol. Technol. 2013, 56, 457-466. [CrossRef]

34. El-Mahdy, R.; Mostafa, A.; El-Kannishy, G. High level aminoglycoside resistant enterococci in hospital-acquired urinary tract infections in Mansoura, Egypt. Germs 2018, 8, 186-190. [CrossRef]

35. Shete, V.; Grover, N.; Kumar, M. Analysis of aminoglycoside modifying enzyme genes responsible for high-level aminoglycoside resistance among enterococcal isolates. J. Pathog. 2017, 2017, 3256952. [CrossRef]

36. Crank, C.W.; O'Driscoll, T. Vancomycin-resistant enterococcal infections: Epidemiology, clinical manifestations, and optimal management. Infect. Drug Resist. 2015, 8, 217-230. [CrossRef]

37. Wright, G.D.; Ladak, P. Overexpression and characterization of the chromosomal aminoglycoside $6^{\prime}$-N-acetyltransferase from Enterococcus faecium. Antimicrob. Agents Chemother. 1997, 41, 956-960. [CrossRef] [PubMed]

38. León-Sampedro, R.; Novais, C.; Peixe, L.; Baquero, F.; Coque, T.M. Diversity and Evolution of the Tn5801-tet(M)-Like Integrative and Conjugative Elements among Enterococcus, Streptococcus, and Staphylococcus. Antimicrob. Agents Chemother. 2016, 60, 1736-1746. [CrossRef] 
39. Mingoia, M.; Morici, E.; Tili, E.; Giovanetti, E.; Montanari, M.P.; Varaldo, P.E. Characterization of Tn5801.Sag, a variant of Staphylococcus aureus Tn916 family transposon Tn5801 that is widespread in clinical isolates of Streptococcus agalactiae. Antimicrob. Agents Chemother. 2013, 57, 4570-4574. [CrossRef] [PubMed]

40. Perin, L.M.; Miranda, R.O.; Todorov, S.D.; Franco, B.D.G.D.M.; Nero, L.A. Virulence, antibiotic resistance and biogenic amines of bacteriocinogenic lactococci and enterococci isolated from goat milk. Int. J. Food Microbiol. 2014, 185, 121-126. [CrossRef] [PubMed] 